\title{
Observational, multicentre study on the epidemiology of haemoptysis
}

To the editor:

Haemoptysis, which is a challenging symptom accounting for $10-15 \%$ of all pulmonology consultations, may be associated with life-threatening medical conditions such as lung cancer [1-7].

Its aetiology and epidemiology vary widely among studies according to geographic locations and time of publication, epidemiological design, and diagnostic tests employed [2-8]. Bronchiectasis, malignancies, post-tuberculosis sequelae, and idiopathic bleedings have been recognised as the most frequent causes of haemoptysis in Europe over the last decade [3-7]. No guidelines exist suggesting an optimal work-up of symptoms, and data on the diagnostic yield of the most commonly prescribed examinations are limited [8].

The aim of this observational, prospective, multicentre study was to investigate the haemoptysis aetiology in association with its severity in an Italian population. We also evaluated the diagnostic yield of the prescribed diagnostic tests.

The study was approved by the ethical committees of five Italian participating hospitals and registered at ClinicalTrials.gov (identifier: NCT02045394). Written informed consent was signed by the recruited patients, who were followed-up for 18 months. Herein, we report the findings of the baseline assessment.

From July 2013 to September 2015, consecutive adult (i.e. $\geqslant 18$ years old) patients with haemoptysis were considered eligible. The following were considered as exclusion criteria: 1) aetiology of haemoptysis already detected (e.g. proven cancer-related and/or bronchiectasis-related haemoptysis); and 2) refusal to sign the informed consent.

Patients were divided into three groups on the basis of the total amount of blood expectorated in $24 \mathrm{~h}$ [2, 3, 9]: mild (i.e. drops of blood to $20 \mathrm{~mL}$ in $24 \mathrm{~h}$ ), moderate (i.e. $20-500 \mathrm{~mL}$ in $24 \mathrm{~h}$ ), and severe (i.e. $>500 \mathrm{~mL}$ in $24 \mathrm{~h}$ ).

All enrolled patients underwent physical examination and blood analysis. Subsequent tests deemed necessary for the diagnosis (i.e. sputum cultures, chest radiography, multi-detector chest computed tomography (CT), bronchoscopy, otorhinolaryngological evaluation, angiography) were chosen by the attending clinician on the basis of the clinical hypothesis and the symptom-driven diagnostic protocols of each hospital involved in the study.

Final diagnosis was established in each centre, on the basis of the clinical and imaging evidence, by a multidisciplinary consensus that involved pulmonologists, radiologists, pathologists and otorhinolaryngologists.

An electronic ad hoc form was created to collect demographic, epidemiological and clinical variables. Absolute and relative frequencies were used to summarise qualitative variables. Quantitative variables, for which the non-parametric distribution was assessed with the Shapiro-Wilk test, were summarised with medians and interquartile ranges (IQRs). 95\% confidence intervals were computed to provide an interval estimation. The statistical software used for all the computations was Stata13.0 (StataCorp, College Station, TX, USA).

During the study period, 606 patients (median age 67 (IQR 52-76) years) were enrolled. More than half (327/606, 54.0\%; 95\% CI 50.0-57.9\%) had a positive smoking history and most of them (404/606, 66.7\%; 95\% CI 62.8-70.3\%) were male. The majority (424/606, 70.0\%; 95\% CI 66.2-73.5\%) had mild epidemiology of haemoptysis. Eur Respir J 2018; 51: 1701813 [https://doi.org/10.1183/13993003.018132017]. 
TABLE 1 Haemoptysis aetiologies related to symptom severity and diagnostic tests prescribed for the assessment of aetiology and their diagnostic yield

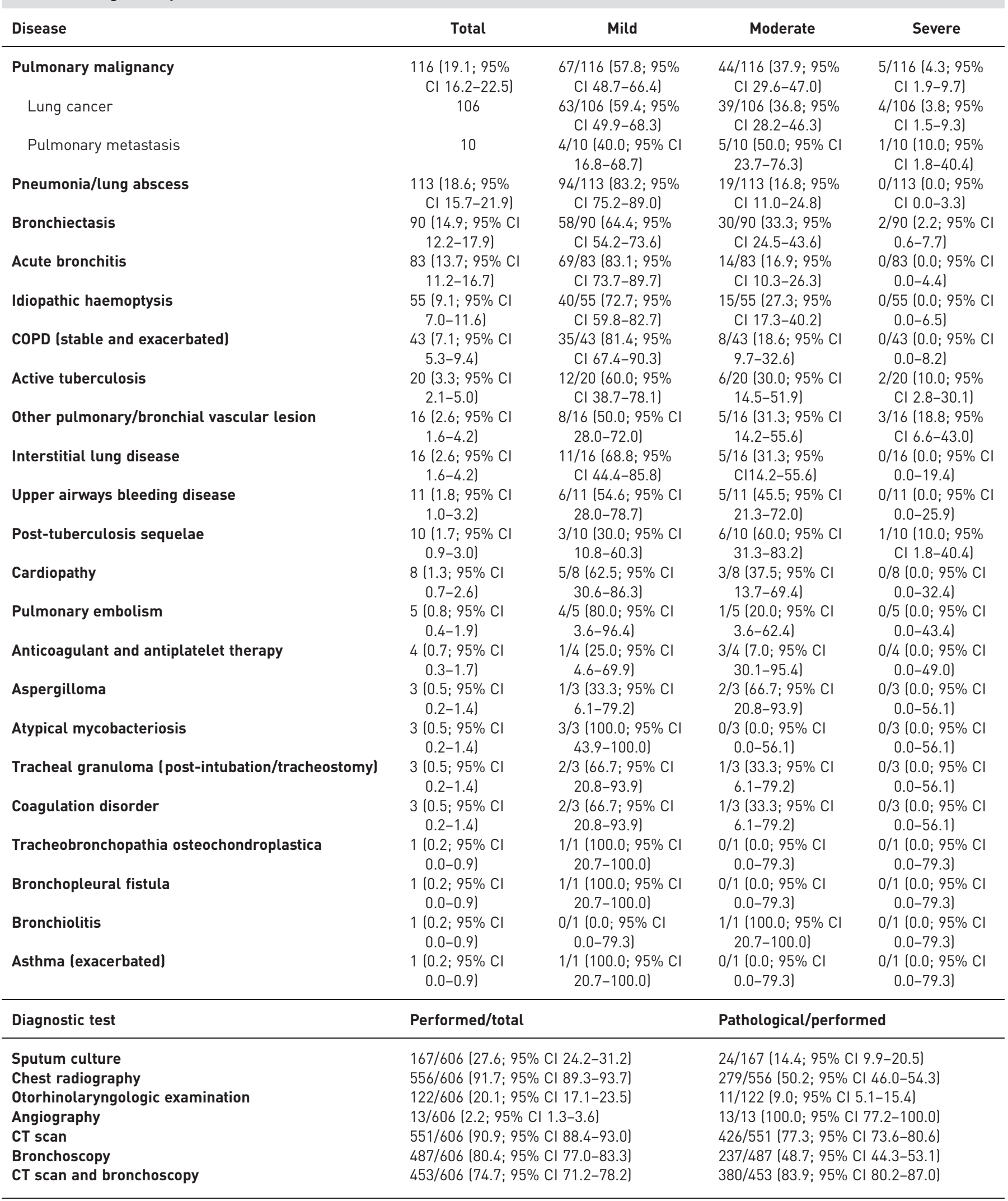

Data are presented as $\mathrm{n}$ or $\mathrm{n}(\%)$. COPD: chronic obstructive pulmonary disease; CT: computed tomography. 
haemoptysis; moderate and severe haemoptysis were recorded in 169/606 (27.9\%; 95\% CI 24.5-31.6\%) and 13/606 (2.2\%; 95\% CI 1.3-3.6\%) patients, respectively.

Pulmonary malignancy (116/606, 19.1\%; 95\% CI 16.2-22.5\%), pneumonia/lung abscess (113/606, 18.6\%; 95\% CI 15.7-21.9\%) and bronchiectasis (90/606, 14.9\%; 95\% CI 12.2-17.9\%) were the most frequent causes of haemoptysis. Idiopathic haemoptysis was diagnosed in only 55/606 (9.1\%; 95\% CI 7.0-11.6\%) patients (table 1).

The majority of neoplasms were lung cancers $(106 / 116,91.3 \%)$ with endobronchial lesions $(84 / 116$, 72.4\%). Malignancies and bronchiectasis were the leading causes of moderate and severe haemoptysis, while pneumonia and acute bronchitis were the most frequent cause of mild bleeding (table 1).

The total number of prescribed diagnostic examinations and their diagnostic yield are shown in table 1. Chest radiography, CT scan and bronchoscopy, which were the most employed tests, had a diagnostic yield of $50.2 \%$ (95\% CI $46.0-54.3 \%$ ), $77.3 \%$ (95\% CI $73.6-80.6 \%$ ) and $48.7 \%$ (95\% CI $44.3-53.1 \%$ ), respectively. The combination of CT and bronchoscopy showed a diagnostic yield of $83.9 \%$ (95\% CI $80.2-$ $87.0 \%)$. The majority of bronchoscopies $(472 / 487,96.5 \%)$ were performed with the flexible instrument, while $13 / 487$ (2.7\%) were performed with the rigid scope.

This is, to our best knowledge, the largest prospective study specifically designed to evaluate the epidemiology of haemoptysis and its severity in a high-income country.

Our results show that pulmonary malignancy is the most frequent (19.1\%) aetiology, with endobronchial lung cancer being the main type. However, pneumonia (18.6\%), bronchiectasis (14.9\%) and acute bronchitis (13.7\%), mostly inducing mild bleeding, are frequent causes of haemoptysis.

Notably, a recent study performed in an Italian emergency department showed similar frequencies for three aetiologies (i.e. malignancy, pneumonia and bronchiectasis) [6]. Moreover, malignancy was the most important case of haemoptysis in a recent study carried out in Turkey [10].

Our results show some differences in comparison with other retrospective studies performed in Europe over the last decade. They showed that bronchiectasis, post-TB sequelae and idiopathic haemoptysis were the most important causes [3-5, 7]. Although malignancies were less frequent than other aetiologies, their percentage $(13 \%-17.8 \%)$ was only slightly lower than that found in our cohort [3-5]. Bronchiectasis and respiratory infections such as pneumonia and acute bronchitis are frequent aetiologies in the latest European studies [3-7]. Bronchiectasis was also the main bleeding cause in recent epidemiological investigations performed in South Korea and Iran [11, 12].

Two European studies investigated the symptom aetiology based on hospital discharge diagnosis codes and found that cryptogenic haemoptysis was the most frequent cause (48.9-52.0\%) [5, 7]. Idiopathic haemoptysis accounted only for $9 \%$ of all causes in our cohort, but its frequency might be slightly over-estimated as the present analysis does not include the follow-up assessment. In comparison with previous studies, we only considered those cases to be idiopathic in which all the examinations, including both radiological and endoscopic, failed to provide a diagnosis [2, 8, 13]. Furthermore, the use of the administrative coding system to estimate the epidemiology of a symptom might have biased their analysis, thus explaining the discrepancy with our data $[5,7]$.

For centuries, haemoptysis has been considered pathognomonic for pulmonary tuberculosis [2, 3]. In our cohort, active tuberculosis accounted only for $3.3 \%$ of all cases, confirming the low incidence recorded in Europe $(0.3-10.0 \%)$ over the past decade [3-7]. In geographical areas with a higher TB incidence, TB remains a relevant cause of haemoptysis (15.0-24.8\%) (e.g. in India it represents $79.2 \%$ of all causes) [11, 12, 14].

In terms of absolute frequency, neoplasms and bronchiectasis, which caused mild bleedings in the majority of the cases, were also the main causes of moderate-to-severe haemoptysis. Bronchiectasis is recognised as the leading cause of severe haemoptysis in recently reported studies $[3,11,12,15]$. Notably, a Greek study, in which patients were classified following similar criteria adopted in our study, reported bronchiectasis as the first cause of moderate-to-severe haemorrhages [3].

It should be highlighted that no guidelines exist on an ideal staging method for the symptom severity. Therefore, we chose threshold values adopted in previous epidemiological studies [2, 3, 9].

Several diagnostic techniques are prescribed to assess the haemoptysis aetiology. Chest radiography, CT and bronchoscopy were the most frequently employed tools. While CT had the highest diagnostic yield, the combination of bronchoscopy and CT (diagnostic yield: 83.9\%) was more effective and relevant than either test alone, confirming their synergistic role $[2,3,11]$.

In conclusion, malignancy, bronchiectasis and pneumonia are the main haemoptysis aetiologies in our Italian cohort. Idiopathic bleeding shows a low incidence. Pneumonia and acute bronchitis are the leading 
aetiologies of mild haemoptysis, while neoplasms and bronchiectasis are leading aetiologies of moderate-to-severe forms. Owing to their complementary role, the combination of bronchoscopy and CT has a high yield in the diagnostic work-up of patients with haemoptysis.

Michele Mondoni ${ }^{1}$, Paolo Carlucci ${ }^{1}$, Sara Job ${ }^{1}$, Elena Maria Parazzini ${ }^{1}$, Giuseppe Cipolla ${ }^{2}$, Matteo Pagani $^{2}$, Francesco Tursi ${ }^{2}$, Luigi Negri ${ }^{2}$, Alessandro Fois ${ }^{3}$, Sara Canu ${ }^{3}$, Antonella $\mathrm{Arcadu}^{3}$, Pietro Pirina ${ }^{3}$, Martina Bonifazi ${ }^{4,5}$, Stefano Gasparini ${ }^{4,5}$, Silvia Marani ${ }^{6}$, Andrea Claudio Comel ${ }^{7}$, Franco Ravenna ${ }^{6}$, Simone Dore ${ }^{8}$, Fausta Alfano ${ }^{1}$, Giuseppe Francesco Sferrazza Papa ${ }^{1,9}$, Fabiano Di Marco ${ }^{1}$, Stefano Centanni ${ }^{1}$ and Giovanni Sotgiu $\oplus^{8}$

${ }^{1}$ Respiratory Unit, ASST Santi Paolo e Carlo, San Paolo Hospital, Department of Scienze della Salute, Università degli Studi di Milano, Milan, Italy. ${ }^{2}$ ASST Lodi, UOC Pneumologia, Lodi, Italy. ${ }^{3}$ Lung Disease Unit, Dept of Clinical and Experimental Medicine, University of Sassari, Sassari, Italy. ${ }^{4}$ Dept of Biomedical Sciences and Public Health, Università Politecnica delle Marche, Ancona, Italy. ${ }^{5}$ Pulmonology Unit, AOU "Ospedali Riuniti”, Ancona, Italy. 'ASST Mantova, Dipartimento Cardio-Toraco-Vascolare, Unità Operativa di Pneumologia e UTIR, Mantova, Italy. ${ }^{7}$ UO Pneumologia, Ospedale Pederzoli, Peschiera del Garda, Italy. ${ }^{8}$ Clinical Epidemiology and Medical Statistics Unit, Dept of Biomedical Sciences, University of Sassari, Sassari, Italy. ${ }^{9}$ Casa di Cura del Policlinico, Dipartimento di Scienze Neuroriabilitative, Milan, Italy.

Correspondence: Michele Mondoni, Respiratory Unit, ASST Santi Paolo e Carlo, San Paolo Hospital, Department of Scienze della Salute, Università degli Studi di Milano; Via Di Rudinì n. 8, 20142, Milan, Italy. E-mail: michele. mondoni@asst-santipaolocarlo.it

Received: Aug 112017 | Accepted after revision: Sept 202017

This study was registered at ClinicalTrials.gov (identifier: NCT02045394).

Conflict of interest: None declared.

\section{References}

1 Lee YJ, Lee SM, Park JS, et al. The clinical implication of bronchoscopy in hemoptysis patients with no explainable lesions in computed tomography. Respir Med 2012; 106: 413-419.

2 Hirshberg B, Biran I, Glazer M, et al. Hemoptysis: etiology, evaluation, and outcome in a tertiary referral hospital. Chest 1997; 112: 440-444.

3 Tsoumakidou M, Chrysofakis G, Tsiligianni I, et al. A prospective analysis of 184 hemoptysis cases - diagnostic impact of chest X-ray, computed tomography, bronchoscopy. Respiration 2006; 73: 808-814.

4 Soares Pires F, Teixeira N, Coelho F, et al. Hemoptysis - etiology, evaluation and treatment in a university hospital. Rev Port Pneumol 2011; 17: 7-14.

5 Abdulmalak C, Cottenet J, Beltramo G, et al. Haemoptysis in adults: a 5-year study using the French nationwide hospital administrative database. Eur Respir J 2015; 46: 503-511.

6 Vanni S, Bianchi S, Bigiarini S, et al. Management of patients presenting with haemoptysis to a Tertiary Care Italian Emergency Department: the Florence Haemoptysis Score (FLHASc). Intern Emerg Med 2017; in press [https://doi.org/10.1007/s11739-017-1618-8].

7 Nielsen K, Gottlieb M, Colella S, et al. Bronchoscopy as a supplement to computed tomography in patients with haemoptysis may be unnecessary. Eur Clin Respir J 2016; 3: 31802.

8 Mondoni M, Sferrazza Papa GF, Sotgiu G, et al. Haemoptysis: a frequent diagnostic challenge. Eur Respir J 2016; 47: $348-350$.

9 Grosu HB, Casal RF, Morice RC, et al. Bronchoscopic findings and bleeding control predict survival in patients with solid malignancies presenting with mild hemoptysis. Ann Am Thorac Soc 2013; 10: 342-349.

10 Uzun O, Atasoy Y, Findik S, et al. A prospective evaluation of hemoptysis cases in a tertiary referral hospital. Clin Respir J 2010; 4: 131-138.

11 Seon HJ, Kim YH, Kwon YS. Localization of bleeding sites in patients with hemoptysis based on their chest computed tomography findings: a retrospective cohort study. BMC Pulm Med 2016; 16: 160.

12 Davoodi M, Kordi M, Gharibvand MM, et al. Hemoptysis: comparison of diagnostic accuracy of multi detector CT scan and bronchoscopy. Glob J Health Sci 2015; 7: 373-377.

13 Herth F, Ernst A, Becker HD. Long-term outcome and lung cancer incidence in patients with hemoptysis of unknown origin. Chest 2001; 120: 1592-1594.

14 Singh SK, Tiwari KK. Etiology of hemoptysis: A retrospective study from a tertiary care hospital from northern Madhya Pradesh, India. Indian J Tuberc 2016; 63: 44-47.

15 Chalumeau-Lemoine L, Khalil A, Prigent H, et al. Impact of multidetector CT-angiography on the emergency management of severe hemoptysis. Eur J Radiol 2013; 82: e742-e747. 